\title{
REVIEW
}

\section{A systematic review of the effectiveness of individual, community and societal-level interventions at reducing socio-economic inequalities in obesity among adults}

\author{
FC Hillier-Brown ${ }^{1,2,3}$, CL Bambra ${ }^{1,3}$, J-M Cairns ${ }^{1,3}$, A Kasim ${ }^{3}$, HJ Moore ${ }^{2,3}$ and CD Summerbell ${ }^{2,3}$
}

BACKGROUND: Socioeconomic inequalities in obesity are well established in high-income countries. There is a lack of evidence of the types of intervention that are effective in reducing these inequalities among adults.

OBJECTIVES: To systematically review studies of the effectiveness of individual, community and societal interventions in reducing socio-economic inequalities in obesity among adults.

METHODS: Nine electronic databases were searched from start date to October 2012 along with website and grey literature searches. The review examined the best available international evidence (both experimental and observational) of interventions at an individual, community and societal level that might reduce inequalities in obesity among adults (aged 18 years or over) in any setting and country. Studies were included if they reported a body fatness-related outcome and if they included a measure of socioeconomic status. Data extraction and quality appraisal were conducted using established mechanisms and narrative synthesis was conducted.

RESULTS: The 'best available' international evidence was provided by 20 studies. At the individual level, there was evidence of the effectiveness of primary care delivered tailored weight loss programmes among deprived groups. Community based behavioural weight loss interventions and community diet clubs (including workplace ones) also had some evidence of effectiveness-at least in the short term. Societal level evaluations were few, low quality and inconclusive. Further, there was little evidence of long term effectiveness, and few studies of men or outside the USA. However, there was no evidence to suggest that interventions increase inequalities.

CONCLUSIONS: The best available international evidence suggests that some individual and community-based interventions may be effective in reducing socio-economic inequalities in obesity among adults in the short term. Further research is required particularly of more complex, multi-faceted and societal-level interventions.

International Journal of Obesity (2014) 38, 1483-1490; doi:10.1038/ijo.2014.75

\section{INTRODUCTION}

In developed countries strong inverse associations between socio-economic indicators and obesity in adulthood have been demonstrated. Cross-sectional evidence continuously shows higher obesity prevalence in disadvantaged adults compared with those who are more affluent ${ }^{1-4}$ although these associations appear to be strongest in women. Longitudinal studies also show that weight gain over time and risk of obesity in later life is greatest in those of lowest socio-economic position. ${ }^{4-6}$ There is also recent evidence to suggest that the disparity in obesity between those of the highest and those of the lowest socioeconomic position may continue to widen in the future. Reducing socio-economic inequalities in health, and risk factors for disease, is a major public health concern; however, in terms of obesity there is a distinct lack of evidence of the types of intervention that are effective in reducing such inequalities. ${ }^{8-10}$ This article aims to systematically review the best available evidence of the effectiveness of interventions (individual, community and societal) in reducing socio-economic inequalities in obesity among adults.
How interventions can impact on inequalities in obesity Interventions can be characterized by their level of action and their approach to tackle inequalities. Whitehead ${ }^{11}$ describes four levels of interventions to tackle inequalities: strengthening individuals (person based strategies to improve the health of disadvantaged individuals), strengthening communities (improving the health of disadvantaged communities and local areas by building social cohesion and mutual support via collective activities), improving living and work environments (reducing exposure to health-damaging material and psychosocial environments across the whole population) and promoting healthy macro-policy (improving the macro-economic, cultural and environmental context that influence the standard of living achieved by the whole population). According to Graham and Kelly, ${ }^{13}$ these interventions are underpinned by one of the three different approaches to health inequality: disadvantage (improving the absolute position of the most disadvantaged individuals and groups), gap (reducing the relative gap between the best and worst off groups), or gradient (reducing the entire social gradient, defined as 'the higher the social position, the

\footnotetext{
${ }^{1}$ Department of Geography, Wolfson Research Institute, Durham University Queen's Campus, Stockton-on-Tees, UK; ${ }^{2}$ School of Medicine, Pharmacy and Health, Wolfson Research Institute, Durham University Queen's Campus, Stockton-on-Tees, UK and ${ }^{3}$ Wolfson Research Institute for Health and Wellbeing, Durham University Queen's Campus, Stockton-onTees, UK. Correspondence: Professor CL Bambra, Department of Geography, Wolfson Research Institute for Health and Wellbeing, Durham University Queen's Campus, Stocktonon-Tees TS17 6BH, UK.

E-mail: clare.bambra@durham.ac.uk

Received 9 December 2013; revised 13 March 2014; accepted 22 March 2014; accepted article preview online 12 May 2014 ; advance online publication, 17 June 2014
} 
better the health' (Marmot and Wilkinson, ${ }^{12}$ (page 2)) Interventions are thus either targeted (directed at those who are disadvantaged) or universal (interventions that influence the entire social gradient).

The aim of this review was to systematically examine the effectiveness of interventions (individual, community and societal) operating via different approaches (targeted or universal) in reducing socio-economic inequalities in obesity among adults. A companion paper examines interventions for reducing socioeconomic inequalities in obesity among children (Hillier-Brown et al., under review).

\section{MATERIALS AND METHODS}

The review was carried out following established criteria for the good conduct and reporting of systematic reviews. ${ }^{14,15}$ The full review protocol is published elsewhere ${ }^{16}$ and is registered with the PROSPERO International Prospective Register of Systematic Reviews (registration number: CRD42013003612). The full review is available to view at http://www.phr. nihr.ac.uk/funded_projects/obesity.asp. ${ }^{17}$

\section{Data sources}

The following electronic databases were searched from the start date up to the 11 October 2012: MEDLINE, EMBASE, CINAHL, PsycINFO, Social Science Citation Index, ASSIA, IBSS, Sociological Abstracts and the NHS Economic Evaluation Database (see additional file for an example search strategy). We did not exclude papers on the basis of language, country or publication date. The electronic database searches were supplemented with website and grey literature searches.

\section{Types of intervention}

Our review examined interventions at the individual, community and societal (environment and macro-policy) level which might reduce inequalities in obesity among adults (aged 18 years or older). We defined individual level interventions as those that included individualized/one-toone health promotion, education, advice, counselling or subsidy and were conducted in a health care or research setting, or in participant's homes. Community level interventions were defined as group-based health promotion, education, advice, counselling or subsidy only interventions, or interventions conducted in a community setting (for example, a workplace, community centre, sports centre and shop). Societal level studies were split into two sub-groups: Societal-environment level interventions, which were defined as those that included a change in environment or access to environment; and Societal-policy level interventions as macro-level policies such as taxation, advertising restriction or subsidies. Interventions were also classified in terms of whether they took a gradient approach and included participants of all socio-economic status (SES) ('universal' interventions) or a targeted approach, that is, aimed at low-SES participants only ('targeted' interventions). Measures and proxy measures of SES were income, education, occupation or area level disadvantage. Interventions that involved drugs or surgery, and laboratorybased studies, were excluded from the review.

Our review considered strategies, which might reduce existing inequalities in the prevalence of obesity (that is, effective targeted interventions or universal interventions that work more effectively in low-SES groups), as well as those interventions, which might prevent the development of inequalities in obesity (that is, universal interventions that work equally along the SES gradient).

\section{Types of studies}

Our review included randomized and non-randomized controlled trials (classified as experimental studies) that included either a non-treatment control group or standard treatment group, and prospective and retrospective cohort studies, with or without control/standard treatment groups, and prospective repeat cross-sectional studies with or without control/standard treatment groups (classified as observational studies). Only studies with duration of at least 12 weeks (combination of intervention and follow up) were included. For the purpose of this article only the best evidence available for each intervention level is reported however, this included randomized and non-randomized controlled trials (experimental studies) as well as uncontrolled prospective cohort studies (observational studies).

\section{Types of outcome measure}

Studies were included if they reported a primary outcome that is a proxy for body fatness (weight and height; body mass index; waist measurement/waist to hip proportion; percentage body fat; skin fold thickness) and if they examined differential effects with regard to socio-economic status or were targeted specifically at disadvantaged groups or were conducted in deprived areas.

\section{Data extraction and quality appraisal}

The initial screening of titles and abstracts was conducted by one reviewer with a random $10 \%$ of the sample checked by a second reviewer. Data extraction was conducted by one reviewer using established data extraction forms and independently checked by a second reviewer. The methodological quality of the included studies was appraised independently by two reviewers using the Cochrane Public Health Review Group recommended Effective Public Health Practice Project Quality Assessment Tool for Quantitative Studies. ${ }^{18}$ Any discrepancies were resolved through discussion between the authors and, if consensus was not reached, with the project lead.

\section{Analysis and synthesis}

Our full review ${ }^{17}$ used very broad study inclusion criteria and conducted a very wide search to capture the entire evidence based on the effects of interventions to reduce inequalities in obesity among adults. We examined the full papers of all studies which fit our population, intervention, design and outcome inclusion criteria, even if there is no mention of socio-economic inequalities in the abstract. By adopting this strategy we were less likely to exclude studies which undertook subgroup analyses by socio-economic status but did not publish the findings in the abstract. This resulted in a very large evidence base that was much larger than anticipated.

To make sense of it for policy and practice, this article focuses only on a narrative synthesis of the 'best available' international evidence for each intervention type. Best available evidence was defined in terms of both study design and study quality by each intervention type. We considered experimental study designs (randomized, including cluster randomized and non-randomized controlled trials) as providing better evidence than observational study designs (prospective and retrospective cohort studies, with or without control/standard treatment groups, and prospective repeat cross-sectional studies with or without control/standard treatment groups), and 'strong' quality studies as providing the best evidence, followed by 'moderate' and then 'weak' quality studies. Only those studies that provided the highest quality evidence for each intervention type are synthesized in this paper.

\section{RESULTS}

Our database searches indentified 70730 records. After title and abstract screening 3142 papers were retreived. Supplementary searching revealed four additional studies that met the inclusion criteria. After full paper screening, the 'best available' evidence for each intervention level was obtained from 20 studies (5 individual level, 12 community, 1 societal-environmental and 2 societalmacro-policy interventions). For the individual and community level interventions, the 'best available' evidence is provided by strong quality, experimental studies (randomized and nonrandomized controlled trials, randomized and non-randomized cluster trials). For the societal-environmental level interventions a moderate quality experimental study was the strongest identified. For the societal-macro-policy interventions only weak quality observational studies (one retrospective uncontrolled cohort study and one serial cross-sectional study) were located and these therefore provide the 'best available' international evidence.

The descriptions and findings of the 'best available' evidence studies are summarized in Tables 1-3. Overall, this shows that the various interventions either reduced inequalities in obesity (that is, they reduced prevalence of obesity-related outcomes among lowSES groups or they closed the SES gap) or had no effect, with no 
Table 1. Summary details of the individual level studies included in the review

\begin{tabular}{|c|c|c|c|c|c|c|c|}
\hline Study & Design \& quality appraisal $^{a}$ & Setting \& participants & Intervention $^{\mathrm{b}}$ & Inequality ${ }^{c}$ & \multicolumn{2}{|c|}{ 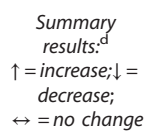 } & $\begin{array}{l}\text { Impact on } \\
\text { inequalities } \\
\text { in obesity }\end{array}$ \\
\hline & \multicolumn{7}{|c|}{ Individual level interventions } \\
\hline Craigie et al. ${ }^{20}$ & $\begin{array}{l}\text { Randomised controlled pilot } \\
\text { study; } 12 \text {-week follow-up } \\
\text { (post-intervention); final } \\
\text { sample = 36; quality= strong }\end{array}$ & $\begin{array}{l}\text { Participant's homes, } \\
\text { UK; } \\
\text { Mean }=30 \text { years; } \\
100 \% \text { female; BMI } \\
>25 \mathrm{~kg} \mathrm{~m}^{-2} \text { ' } \\
6-18 \text { months post- } \\
\text { partum }\end{array}$ & $\begin{array}{l}\text { 12-week nutrition and physical activity treatment } \\
\text { intervention: post-partum weight loss programme } \\
\text { (WeighWell)- face-to-face consultations }(\times 3) \text { and } \\
\text { telephone support (minimum } \times 3 \text { ) from trained } \\
\text { lifestyle counsellor. Motivational interviewing } \\
\text { techniques, calorie reduced diet and physical activity } \\
\text { goals; no cost data reported }\end{array}$ & $\begin{array}{l}\text { Disadvantage: women } \\
\text { living in areas of moderate } \\
\text { to high deprivation }\end{array}$ & $\begin{array}{l}\text { Body } \\
\text { weight } \\
\text { BMl } \\
\% \text { body } \\
\text { fat } \\
\text { WC }\end{array}$ & $\begin{array}{l}\downarrow \\
\downarrow \\
\downarrow \\
\leftrightarrow\end{array}$ & + \\
\hline Davis Martin et al. ${ }^{19}$ & $\begin{array}{l}\text { Randomised control trial; } \\
6 \text { month follow-up (post- } \\
\text { intervention; final } \\
\text { sample =106; quality = strong }\end{array}$ & $\begin{array}{l}\text { Two medical centres, } \\
\text { USA; } \\
18-62 \text { years; } \\
100 \% \text { female; } \\
\text { overweight and } \\
\text { obese }\end{array}$ & $\begin{array}{l}6 \text { month nutrition and physical activity treatment } \\
\text { intervention: tailored and culturally appropriate } \\
\text { weight management programme - physician } \\
\text { delivered }(1 \times 15 \text { min consultation per month), } \\
\text { individual recommendations and strategies } \\
\text { provided by health psychologist, dietitian and } \\
\text { exercise physiologist; no cost data reported }\end{array}$ & $\begin{array}{l}\text { Disadvantage: low income, } \\
\text { African-American women }\end{array}$ & $\begin{array}{l}\text { Body } \\
\text { weight }\end{array}$ & $\downarrow$ & + \\
\hline Whittemore et al. ${ }^{21}$ & $\begin{array}{l}\text { Cluster randomized controlled } \\
\text { pilot study; } 6 \text { month follow-up } \\
\text { (post- intervention); final } \\
\text { sample = 51; quality = strong }\end{array}$ & $\begin{array}{l}\text { Four health care } \\
\text { practices, USA; } 92 \% \\
\text { female; mean age } \approx 46 \\
\text { years; } \\
\text { BMI } \geqslant 25 \mathrm{~kg} \mathrm{~m}^{-2}\end{array}$ & $\begin{array}{l}6 \text { month nutrition and physical activity treatment } \\
\text { intervention: diabetes prevention programme for } \\
\text { overweight and obese adults-individual sessions } \\
\text { with nurse }(1 \times 30 \mathrm{~min}) \text { and nutritionist }(1 \times 45 \mathrm{~min}) \text {; } \\
\text { culturally relevant education on nutrition, exercise; } \\
\text { behavioural support in identifying goals and } \\
\text { problem-solving barriers to change; motivational } \\
\text { interviewing; no cost data reported }\end{array}$ & $\begin{array}{l}\text { Disadvantage: participants } \\
\text { had moderately low } \\
\text { incomes }\end{array}$ & $\begin{array}{l}\text { Body } \\
\text { weight } \\
\text { BMI } \\
\text { WC }\end{array}$ & $\begin{array}{l}\downarrow \\
\leftrightarrow \\
\leftrightarrow\end{array}$ & 0 \\
\hline Jeffery and French ${ }^{23,24}$ & $\begin{array}{l}\text { Randomised controlled trial; } \\
3 \text { year follow-up (post- } \\
\text { intervention); } \\
\text { final sample }=809 ; \\
\text { quality = strong }\end{array}$ & $\begin{array}{l}\text { Homes, USA; } \\
20-45 \text { years; } \\
100 \% \text { female }\end{array}$ & $\begin{array}{l}3 \text { year nutrition and physical activity prevention } \\
\text { intervention (Pound of Prevention Study): diet and } \\
\text { physical activity education with or without a lottery } \\
\text { incentive; additional voluntary activities (e.g. group } \\
\text { sessions and dance classes); no cost data reported }\end{array}$ & $\begin{array}{l}\text { Gradient: no interaction } \\
\text { between intervention and } \\
\text { participant type (low- } \\
\text { income or high-income } \\
\text { women) }\end{array}$ & $\begin{array}{l}\text { Body } \\
\text { weight }\end{array}$ & $\leftrightarrow$ & 0 \\
\hline Martin et al. ${ }^{22}$ & $\begin{array}{l}\text { Randomised controlled trial; } \\
18 \text { month follow-up } \\
\text { ( } 12 \text { months post-intervention); } \\
\text { final sample }=86 ; \\
\text { quality = strong }\end{array}$ & $\begin{array}{l}\text { Primary care setting, } \\
\text { USA; } 100 \% \text { female; } \\
18-65 \text { years; } \\
\text { overweight or obese } \\
\left(B M I>25 \mathrm{~kg} \mathrm{~m}^{-2}\right)\end{array}$ & $\begin{array}{l}6 \text { month nutrition and physical activity treatment } \\
\text { intervention: tailored weight loss intervention; } \\
\text { monthly counselling sessions; topics included } \\
\text { weight loss, decreasing dietary fat, increasing } \\
\text { physical activity, barriers to weight loss and healthy } \\
\text { alternatives when eating out and shopping; } \$ 35 \\
\text { reimbursement per participant }\end{array}$ & $\begin{array}{l}\text { Disadvantage: low-income } \\
\text { minority women }\end{array}$ & $\begin{array}{l}\text { Weight } \\
\text { loss }\end{array}$ & $\leftrightarrow$ & 0 \\
\hline
\end{tabular}

Abbreviations: BMI, body mass index; EPHPP, effective public health practice project; SES, socio-economic status; WC, waist circumference. ${ }^{\mathrm{a}} \mathrm{Global}$ quality appraisal from EPHPP. ${ }^{18}$ brevention or treatment intervention. ${ }^{c}$ Disadvantage/gradient approach to inequality . ${ }^{d} P<0.05$. This is the relative mean differences between intervention and control at longest follow-up. ${ }^{e}+$, positive intervention effect so it reduces obesity-related outcomes in low-SES groups or reduces the SES gradient in obesity-related outcomes; 0, no intervention effect or no effect on SES gradient in obesity-related outcomes.

studies reporting a negative impact (that is, it increased the gap in obesity-related outcomes). Summaries of each study and their overall impact on SES inequalities in obesity are reported in the tables.

Individual $(n=5)$

Four of the five 'best evidence' individual level studies were from the USA, with the remaining study from the UK. All of the studies had all women or majority women participant groups.

Four of the studies examined tailored weight loss programmes delivered via primary care for low-income groups (targeted approach). One was a randomized controlled trial ${ }^{19}$ of 106 lowincome African-American women that found that after a 6 month intervention, the intervention group demonstrated a significant weight loss $(-2.0 \mathrm{~kg}$; s.d. $=3.2)$ compared with the control group $(+0.2 \mathrm{~kg} ;$ s.d. $=2.9) \quad(P=0.03)$. A small $(n=36)$ randomized controlled pilot study of overweight post-partum women living in areas of moderate to high deprivation in the $U^{20}$ found that after a 12-week intervention, body weight loss was significantly greater in the intervention compared with the comparison group $(-1.6 \mathrm{~kg}$ vs $0.2 \mathrm{~kg} ; P=0.018)$, with significant improvements in BMI $(-0.7$ $\mathrm{kg} \mathrm{m}^{-2}$ vs $\left.0.1 \mathrm{~kg} \mathrm{~m}^{-2} ; P=0.009\right)$ and percentage body fat $(-1.5 \mathrm{vs}$ $-0.5 ; P=0.029$ ) too. There were no significant differences in waist circumference or physical activity. A cluster randomized controlled pilot study ${ }^{21}$ investigated the effects of a 6 month tailored weight loss programme for 51 low-income adults (majority women) considered at high risk of diabetes in four areas of the USA. Post intervention, $25 \%$ of the intervention group achieved a clinically significant weight loss compared with only $11 \%$ of the control group. However, physical activity and nutritional changes were similar among control and intervention groups. Intention-to-treat analysis from another randomized controlled trial ${ }^{22}$ investigating the effects of a 6 month tailored weight loss intervention showed that after 9 months (3 months post-intervention), weight loss among 86 low-income African-American women was significantly higher in the intervention group than the control $(-1.52 \pm 3.72 \mathrm{~kg}$ vs $0.61 \pm 3.37 \mathrm{~kg} ; \mathrm{F}=12.32 ; P<0.01)$ although this was not sustained at 12 or 18 months $(F=0.85, P=0.39)$.

One randomized controlled trial ${ }^{23,24}$ investigated the effects of a 3 year weight gain prevention educational intervention (consisting of monthly newsletters with healthy behaviour messages) among low- and high-income women in the USA (universal approach). After 1 year the intervention improved weight among high-income women only, but after 3 years, there was no significant intervention effect on weight for either high or low-income groups.

\section{Community $(n=12)$}

The vast majority of the 'best evidence' community level studies were from the USA (one each from the UK, Australia and Turkey) and all except three studies had only women participants or a female majority, some had very small sample sizes as they were pilots and many had active controls (usually standard care using individual level interventions).

Eight studies examined community-based group health education and counselling interventions. Two of these examined behavioural weight loss programmes; three examined group lifestyle counselling interventions; three evaluated communitybased group-based health education interventions; and one study 
Table 2. Summary details of the community level studies included in the review

\begin{tabular}{|c|c|c|c|c|c|c|c|}
\hline Study & Design \& quality appraisal $^{a}$ & $\begin{array}{l}\text { Setting \& } \\
\text { participants }\end{array}$ & Intervention $^{\mathrm{b}}$ & 'Inequality & $\begin{array}{l}\text { Summary results: }^{\text {d }} \\
\uparrow=\text { increase } ;=\text { = decrease; } \\
\leftrightarrow=\text { o change }\end{array}$ & & $\begin{array}{l}\text { Impact on } \\
\text { inequalities } \\
\text { in obesity }\end{array}$ \\
\hline $\begin{array}{l}\text { Erfurt } \\
\text { et } a l^{35}\end{array}$ & $\begin{array}{l}\text { Cluster randomized controlled } \\
\text { trial; } 3 \text { year follow-up (post- } \\
\text { intervention); final } \\
\text { sample =1883; quality = strong }\end{array}$ & $\begin{array}{l}4 \text { Workplaces, USA; } \\
39-43 \text { years; } \\
\text { predominately } \\
\text { male }\end{array}$ & $\begin{array}{l}3 \text { year nutrition and physical activity prevention } \\
\text { intervention: screening only (control) vs } \\
\text { screening+health education (A) vs screening } \\
\text { +health education+follow-up counselling (B) vs } \\
\text { screening+health education+follow-up } \\
\text { counselling+organized activities (C); cost of full } \\
\text { group programmes ranged from } 50 \text { to } \$ 100 \text { per } \\
\text { participant }\end{array}$ & $\begin{array}{l}\text { Disadvantage: } \\
\text { manufacturing worksites } \\
\text { (predominately blue collar } \\
\text { employees) }\end{array}$ & $\begin{array}{l}\text { Body weight } \\
\text { All overweight employees } \\
(n=690) \text { : } \\
\text { Intervention A } \\
\text { Intervention B } \\
\text { Intervention C } \\
\text {; Participants of weight } \\
\text { loss components } \\
(n=238) \text { : } \\
\text { Intervention A } \\
\text { Intervention B } \\
\text { Intervention C }\end{array}$ & $\begin{array}{l}\leftrightarrow \\
\leftrightarrow \\
\downarrow \\
\leftrightarrow \\
\downarrow \\
\downarrow\end{array}$ & + \\
\hline $\begin{array}{l}\text { Ockene } \\
\text { et al. }\end{array}$ & $\begin{array}{l}\text { Randomised controlled trial; } 1 \\
\text { year follow-up (post- } \\
\text { intervention); final } \\
\text { sample=288; quality= strong }\end{array}$ & $\begin{array}{l}\text { Senior community } \\
\text { centre, USA; } \\
\text { mean age } \approx 52 \\
\text { years; } \approx 74 \% \text { female }\end{array}$ & $\begin{array}{l}1 \text { year nutrition and physical activity prevention } \\
\text { intervention (Lawrence Latino Diabetes } \\
\text { Prevention Programme): community-based, } \\
\text { literacy-sensitive \& culturally tailored } \\
\text { intervention; individual \& group counselling } \\
\text { sessions: } \$ 661 \text { per participant for intervention } \\
\text { (\$1399 per participant for standard care) }\end{array}$ & $\begin{array}{l}\text { Disadvantage: low-SES } \\
\text { area }\end{array}$ & $\begin{array}{l}\text { Weight } \\
\text { BMI }\end{array}$ & $\downarrow$ & + \\
\hline $\begin{array}{l}\text { Kisioglu } \\
\text { et } \text { al. }^{33}\end{array}$ & $\begin{array}{l}\text { Randomised controlled trial; } \\
6 \text { month follow-up } \\
\text { (approximately } 5 \text { months post- } \\
\text { intervention); final } \\
\text { sample =400; quality = strong }\end{array}$ & $\begin{array}{l}\text { Setting unclear, } \\
\text { Turkey; mean } \\
\text { age = } 34 \text { years; } \\
100 \% \text { women }\end{array}$ & $\begin{array}{l}25 \text {-day nutrition and physical activity prevention } \\
\text { intervention: health training support, nutrition } \\
\text { educational material; encouragement to } \\
\text { participate in education programme; no cost data } \\
\text { reported }\end{array}$ & Disadvantage: low SES & BMI & $\downarrow$ & + \\
\hline $\begin{array}{l}\text { Faucher }^{30} \text {; } \\
\text { Faucher } \\
\text { and } \\
\text { Mobley }^{31}\end{array}$ & $\begin{array}{l}\text { Randomised controlled pilot } \\
\text { trial; } 5 \text { month follow-up (post- } \\
\text { intervention); final sample }=19 ; \\
\text { quality = strong }\end{array}$ & $\begin{array}{l}\text { Community centre, } \\
\text { USA; } 100 \% \text { women; } \\
\text { mean age }=35 \\
\text { years; } \mathrm{BMI} \geqslant 25 \\
\text { obese }\end{array}$ & $\begin{array}{l}\text { 20week nutrition treatment intervention: aimed } \\
\text { at portion control; culturally sensitive and foods } \\
\text { prepared culturally/economically specific to low- } \\
\text { income Mexican-American families; no cost data } \\
\text { reported }\end{array}$ & $\begin{array}{l}\text { Disadvantage: low SES by } \\
\text { income }\end{array}$ & Weight loss & $\downarrow(\mathrm{CS})$ & 0 \\
\hline Rickel $^{29}$ & $\begin{array}{l}\text { Randomised controlled trial; } \\
12 \text { month follow-up (post- } \\
\text { intervention); final } \\
\text { sample=224; quality= strong }\end{array}$ & $\begin{array}{l}\text { Cooperative } \\
\text { extension service } \\
\text { offices, USA; } 50-75 \\
\text { years; } 100 \% \text { female; } \\
\text { BMI } \geqslant 30\end{array}$ & $\begin{array}{l}12 \text { month extended care nutrition and physical } \\
\text { activity treatment intervention: extended care } \\
\text { after a culturally tailored lifestyle intervention - } \\
\text { face-to-face or telephone contact; no cost data } \\
\text { reported }\end{array}$ & $\begin{array}{l}\text { Disadvantage: counties of } \\
\text { low levels of educational } \\
\text { attainment and low } \\
\text { household incomes }\end{array}$ & $\begin{array}{l}\text { Body weight (Caucasians) } \\
\text { Body weight (AA) } \\
\text { Both intervention } \\
\text { conditions vs control (no } \\
\text { differences in weight } \\
\text { change between } \\
\text { intervention groups) }\end{array}$ & $\begin{array}{l}\downarrow \\
\leftrightarrow\end{array}$ & 0 \\
\hline $\begin{array}{l}\text { Auslander } \\
\text { et } \text { al. }^{32}\end{array}$ & $\begin{array}{l}\text { Randomised controlled trial; 3- } \\
\text { month follow-up (post- } \\
\text { intervention); final } \\
\text { sample=239; quality = strong }\end{array}$ & $\begin{array}{l}\text { Community, USA; } \\
25-55 \text { years; } 100 \% \\
\text { female; obese }\end{array}$ & $\begin{array}{l}\text { 3-month nutrition treatment intervention } \\
\text { (Eat Well Live Well): peer education focussing on } \\
\text { nutrition skills tailored to individuals stage of } \\
\text { change; social support from group sessions; no } \\
\text { cost data reported }\end{array}$ & $\begin{array}{l}\text { Disadvantage: low-income } \\
\text { African-American women }\end{array}$ & $\begin{array}{l}\text { BMI } \\
\text { Body weight }\end{array}$ & $\stackrel{\leftrightarrow}{\leftrightarrow}$ & 0 \\
\hline $\begin{array}{l}\text { Baron } \\
\text { et } \mathrm{al.}^{34}\end{array}$ & $\begin{array}{l}\text { Randomised controlled trial; } 1 \\
\text { year follow-up ( } 9 \text { months post- } \\
\text { intervention; final } \\
\text { sample=119; quality= strong }\end{array}$ & $\begin{array}{l}\text { Diet clubs, UK; } 16- \\
70 \text { years; } 85 \% \\
\text { female; overweight }\end{array}$ & $\begin{array}{l}\text { 3-month nutrition treatment intervention: diet } \\
\text { clubs with weekly group meetings; followed } \\
\text { either a LCD or LFD; no cost data reported }\end{array}$ & $\begin{array}{l}\text { Gradient: LCD more } \\
\text { effective than LFD in lower } \\
\text { SES at } 3 \text { months but this } \\
\text { effect was not observed at } \\
1 \text { year }\end{array}$ & Body weight & $\leftrightarrow$ & 0 \\
\hline $\begin{array}{l}\text { Befort } \\
\text { et } a l^{26}\end{array}$ & $\begin{array}{l}\text { Randomised controlled pilot } \\
\text { study; } 16 \text { week follow-up (post- } \\
\text { intervention); final sample = } 33 \text {; } \\
\text { quality = strong }\end{array}$ & $\begin{array}{l}\text { Community health } \\
\text { centre, USA; } \geqslant 18 \\
\text { years; } 100 \% \text { female; } \\
\text { obese } \\
(\mathrm{BMI}=30-50)\end{array}$ & $\begin{array}{l}\text { 16week nutrition and physical activity treatment } \\
\text { intervention: culturally appropriate behavioural } \\
\text { weight loss programme plus motivational } \\
\text { interviewing or health education; calorie reduced } \\
\text { diet; self-monitoring; and food and physical } \\
\text { activity guidance; no cost data reported }\end{array}$ & $\begin{array}{l}\text { Disadvantage: lower } \\
\text { income African-American } \\
\text { women }\end{array}$ & $\begin{array}{l}\text { Body weight } \\
\text { BMI }\end{array}$ & $\stackrel{\leftrightarrow}{\leftrightarrow}$ & 0 \\
\hline $\begin{array}{l}\text { Campbell } \\
\text { et al. }{ }^{36}\end{array}$ & $\begin{array}{l}\text { Cluster randomized controlled } \\
\text { trial; } 18 \text { month follow-up (mid- } \\
\text { intervention); final } \\
\text { sample=538; quality= strong }\end{array}$ & $\begin{array}{l}9 \text { worksites, USA; } \\
100 \% \text { women }\end{array}$ & $\begin{array}{l}\text { 5-year nutrition and physical activity prevention } \\
\text { intervention (health works for women): } \\
\text { individualized computer-tailored health } \\
\text { messages; a natural helpers programme at the } \\
\text { workplace (lay health advisor); no cost data } \\
\text { reported }\end{array}$ & $\begin{array}{l}\text { Disadvantage: low-SES } \\
\text { workplaces }\end{array}$ & BMI & $\leftrightarrow$ & 0 \\
\hline $\begin{array}{l}\text { Howard- } \\
\text { Pitney } \\
\text { et al. }\end{array}$ & $\begin{array}{l}\text { Randomised controlled trial; } \\
\text { 18week follow-up (post- } \\
\text { intervention); final } \\
\text { sample=242; quality = strong }\end{array}$ & $\begin{array}{l}\text { Community setting, } \\
\text { USA; mean age }=31 \\
\text { years; } 85 \% \text { female }\end{array}$ & $\begin{array}{l}\text { 18week nutrition prevention intervention: } \\
6 \text {-week, classroom-based intervention followed } \\
\text { by a } 12 \text {-week maintenance intervention; } \\
\text { culturally sensitive; nutrition education delivered } \\
\text { by professional nutrition health educators; } \\
\text { telephone contact; no cost data reported }\end{array}$ & $\begin{array}{l}\text { Disadvantage: low-income } \\
\text { population }\end{array}$ & BMI & $\leftrightarrow$ & 0 \\
\hline $\begin{array}{l}\text { Olvera } \\
\text { et } a l^{37}\end{array}$ & $\begin{array}{l}\text { Randomised controlled trial; } \\
\text { 12-week follow-up (post- } \\
\text { intervention); final sample }=35 \\
\text { quality = strong }\end{array}$ & $\begin{array}{l}\text { Community } \\
\text { locations, USA; } \\
\text { mean age } \approx 35 \\
\text { years; } 100 \% \text { female } \\
\text { (mothers) }\end{array}$ & $\begin{array}{l}\text { 12-week nutrition and physical activity } \\
\text { prevention intervention: group aerobic or sports } \\
\text { sessions or free play recreational activities; } \\
\text { nutrition sessions; behavioural counselling } \\
\text { sessions; no cost data reported }\end{array}$ & $\begin{array}{l}\text { Disadvantage: low-income } \\
\text { women }\end{array}$ & BMI & $\leftrightarrow$ & 0 \\
\hline $\begin{array}{l}\text { Reid } \\
\text { et al. }\end{array}$ & $\begin{array}{l}\text { Randomised controlled trial; } \\
6 \text { month follow-up (6months } \\
\text { post-intervention); final } \\
\text { sample =149; quality = strong }\end{array}$ & $\begin{array}{l}\text { Community health } \\
\text { centre, Australia; } \\
\approx 40 \% \text { female; mean } \\
\text { age }=41 \text { years }\end{array}$ & $\begin{array}{l}\text { Single session nutrition prevention intervention: } \\
\text { group counselling sessions conducted by a } \\
\text { trained community health nurse; covered } \\
\text { smoking cessation, dietary modification and non- } \\
\text { pharmacological lowering of blood pressure; no } \\
\text { cost data reported }\end{array}$ & $\begin{array}{l}\text { Disadvantage: low-SES } \\
\text { area }\end{array}$ & Weight & $\leftrightarrow$ & 0 \\
\hline $\begin{array}{l}\text { Wing and } \\
\text { Jeffery }{ }^{25}\end{array}$ & $\begin{array}{l}\text { Randomised controlled trial; } \\
10 \text { month follow-up (6months } \\
\text { post-intervention); final } \\
\text { sample =136; quality = strong }\end{array}$ & $\begin{array}{l}\text { Setting unclear, } \\
\text { USA; } 22-55 \text { year } \\
\text { olds; } \approx 50 \% \text { female }\end{array}$ & $\begin{array}{l}\text { 4month nutrition and physical activity treatment } \\
\text { intervention: } 16 \text { weekly group meetings led by a } \\
\text { behaviour therapist and/or a nutritionist; weigh } \\
\text { ins, review of self-monitoring records; lecture or } \\
\text { discussion period; behaviour techniques; no cost } \\
\text { data reported }\end{array}$ & $\begin{array}{l}\text { Gradient: employment did } \\
\text { not affect overall weight } \\
\text { loss }\end{array}$ & Weight & $\leftrightarrow$ & 0 \\
\hline
\end{tabular}

Abbreviations: BMI, body mass index; CS, clinically significant; EPHPP, effective public health practice project; LCD, low carbohydrate diet; LFD, low fat diet; SES, socio-economic status. ${ }^{a} \mathrm{Global}$ quality appraisal from EPHPP. ${ }^{18}$ bPrevention or treatment intervention. 'Disadvantage/gradient approach to inequality. ${ }^{\mathrm{d}} P<0.05$. This is the relative mean differences between intervention and control at the longest follow-up. ${ }^{{ }^{+}}+$, positive intervention effect so it reduces obesityrelated outcomes in low-SES groups or reduces the SES gradient in obesity-related outcomes; 0, no intervention effect or no effect on SES gradient in obesityrelated outcomes. 


\begin{tabular}{|c|c|c|c|c|c|c|c|}
\hline Study & $\begin{array}{l}\text { Design and quality } \\
\text { appraisal }^{\text {a }}\end{array}$ & $\begin{array}{l}\text { Setting \& } \\
\text { participants }\end{array}$ & 'Intervention ${ }^{\mathrm{b}}$ & Inequality $^{c}$ & $\begin{array}{c}\text { Summary } \\
\text { results: } \\
\uparrow=\text { increase; } \downarrow= \\
\text { decrease; } \\
\leftrightarrow=\text { no change }\end{array}$ & & $\begin{array}{l}\text { Impact on } \\
\text { inequalities } \\
\text { in obesity }\end{array}$ \\
\hline $\begin{array}{l}\text { Lemon } \\
\text { et } a l^{39}\end{array}$ & $\begin{array}{l}\text { Cluster randomized } \\
\text { controlled trial; } 24 \text {-month } \\
\text { follow-up (post- } \\
\text { intervention); final } \\
\text { sample =648; } \\
\text { quality = moderate }\end{array}$ & $\begin{array}{l}6 \text { hospital } \\
\text { worksites, } \\
\text { USA; } 18-65 \\
\text { years; } \approx 80 \% \\
\text { female }\end{array}$ & $\begin{array}{l}\text { Environmental interventic } \\
\text { 2-year nutrition and physical } \\
\text { activity prevention intervention: } \\
\text { social marketing campaign, } \\
\text { environmental strategies } \\
\text { promoting physical activity, } \\
\text { environmental strategies } \\
\text { promoting healthy eating, and } \\
\text { strategies promoting } \\
\text { interpersonal support; no cost } \\
\text { data reported }\end{array}$ & $\begin{array}{l}\text { Ons } \\
\text { Gradient: the group } \\
\text { most likely to prevent } \\
\text { weight gain was } \\
\text { those of higher } \\
\text { educational status. }\end{array}$ & BMI & $\leftrightarrow$ & - \\
\hline Kaushal $^{41}$ & $\begin{array}{l}\text { Serial cross-sectional study } \\
\text { (natural study); 8-year } \\
\text { follow-up (post- } \\
\text { intervention); final } \\
\text { sample =68318; } \\
\text { quality = weak }\end{array}$ & $\begin{array}{l}\text { Population } \\
\text { wide, USA; } \\
21-54 \text { years; } \\
26 \% \text { female }\end{array}$ & $\begin{array}{l}\text { Nutrition prevention intervention } \\
\text { (Food Stamp Programme): } \\
\text { investigation of } 1996 \text { federal law } \\
\text { change denying immigrants } \\
\text { access to the programme; no cost } \\
\text { data reported }\end{array}$ & $\begin{array}{l}\text { Disadvantage: low } \\
\text { educated (proxy for } \\
\text { low income) }\end{array}$ & $\begin{array}{l}\text { BMl: } \\
\text { Women } \\
\text { Unmarried } \\
\text { Mothers } \\
\text { Men }\end{array}$ & $\begin{array}{l}\stackrel{\leftrightarrow}{\leftrightarrow} \\
\leftrightarrow\end{array}$ & 0 \\
\hline
\end{tabular}

examined a community-based diet club. The two behavioural weight loss programme studies were conducted among lowincome men and women in the USA (targeted approach). $A$ randomized controlled trial conducted ${ }^{25}$ compared the effects on weight loss of a professionally delivered behavioural therapy active control (for example, problem solving, assertion, stimulus control) with a 4 month behavioural therapy and social support intervention among 136 low-income men and women in the USA. The intervention showed short-term weight loss (post-intervention) but this was not sustained at 7 and 10 month follow-up ( 3 and 6 months post-intervention). A small randomized controlled pilot study ${ }^{26}$ investigated the effects of a 16 week culturallyadapted weekly behavioural weight loss group programme with motivational interviewing vs a behavioural weight loss group programme plus health education (active control) in 33 lower income African-American women (targeted approach). There were significant positive effects post-intervention on weight loss, calorie intake, percentage calories from fat and fruit and vegetable servings per day in both groups. Together these findings suggest that such interventions have short-term (4 months) but not long term (7 months) positive effects on weight loss.

The three studies that examined group lifestyle counselling interventions compared with active controls were conducted among low-SES populations (targeted approach). One found a short-term effect on weight loss among low-income Latino women while the other two found no intervention effect. A randomized controlled trial ${ }^{27}$ evaluated a 1 year communitybased, culturally tailored group lifestyle counselling intervention among 288 low-income (majority female) Latinos in the USA. The intervention group lost significantly more weight postintervention compared with the control group participants (Intervention effect: $-2.5 \mathrm{lb} ; P=0.04$ ) with a significant decrease in BMI $(-0.46 ; P=0.04)$. A randomized controlled trial ${ }^{28}$ in a lowincome urban area of Australia examined the intervention effects on 149 participants of a one-off group lifestyle counselling session (lasting $2 \mathrm{~h}$ ) on the reduction of cardiovascular risk factors among those at high risk (majority male participants). The control group received a pamphlet about reducing cardiovascular risk factors. After 6 months post-intervention, there were no significant weight changes either between or within groups. A randomized controlled trial ${ }^{29}$ investigated a 12 month extended care group counselling intervention (face-to-face group sessions vs individual telephone delivered) compared with an education-only control condition following a weight loss intervention for 224 obese women living in low-SES areas of the USA. The interventions had no overall effect on weight regain compared with the control group.

The three studies that evaluated community-based groupbased health education interventions also followed targeted approaches. A small randomized controlled pilot study ${ }^{30,31}$ compared a 20 week community centre delivered, culturallyadapted group nutritional education intervention against individual-counselling (active control) on weight loss among 19 low-income Mexican-American women in the USA. There were no significant differences in weight loss in the intervention group compared with the control after post-intervention (2.9 kg vs $1.3 \mathrm{~kg}$, 
$P=0.47)$. A randomized controlled trial ${ }^{32}$ investigated the effects of a 3-month peer-educator led group-based dietary skills development intervention for 239 obese low-income AfricanAmerican women in the USA. The control group received a selfhelp workbook and a half-day workshop. There were no significant differences between intervention and control groups for changes in $\mathrm{BMI}$ or weight post-intervention. A randomized controlled trial ${ }^{33}$ examined the effects of a 25-day group-based health education intervention among 400 women from a low-income area of Turkey. The intervention group reported significant changes in lifestyle behaviours leading to reductions in $\mathrm{BMI}(\mathrm{P}=0.014)$ and an increase in those with an ideal BMI ( 86 vs $60, P=0.009$ ) after 6 months ( 5 months post-intervention; no post-intervention measures were taken).

The final community-based group health education and counselling intervention study was a small randomized controlled trial that took a universal approach. ${ }^{34}$ It compared the effects by social class status of weekly community diet clubs in the UK that used two different weight reducing diets: low carbohydrate compared with low fat/high fibre. Moderate weight losses were observed among 119 participants (majority women) in both diet groups after 3 months (post-intervention) but there was no significant difference at 1 year follow-up. Both diets worked equally well among the higher SES participants (social class I or II). Among the lower SES participants (III-V) the low carbohydrate diet was particularly effective at 3 months (post-intervention) (weight loss $=5.0 \mathrm{~kg}$ vs $3.0 \mathrm{~kg} ; 95 \% \mathrm{Cl}$ of difference $=0.3-3.9$ ) but this effect was lost at 1 year.

Two community level studies examined workplace delivered group interventions with divergent results. One study of blue collar male workers in the USA found positive long term effects ( 3 years) of an extensive and complex health promotion, counselling and physical activity intervention on weight loss and weight maintenance. A study of blue collar women employees found no effect on BMI of a lay health advisor programme over 5 years. A cluster randomized trial ${ }^{35}$ investigated the effects of four 3 year wellbeing interventions (active control condition, health screening only; intervention $A$, health screening and health education; intervention $B$, health screening, health education and follow-up counselling; or intervention $C$, health screening, health education, follow-up counselling and organized workplace activities) among 690 overweight (majority male), blue collar workers across four manufacturing worksites in the USA. After the 3 years, there was a significant intervention effect as the control group gained weight, intervention groups $A$ and $B$ experienced no weight change and intervention group $C$ lost weight $(P<0.001)$. Another cluster randomized controlled trial conducted by Campbell et al. ${ }^{36}$ examined a 5-year workplace health promotion programme among 859 low skill women workers in the USA. The intervention consisted of two strategies: (1) individualized computer-tailored health messages; and (2) a lay health advisors programme. Data were collected at 6 and 18 months during the programme and there were no significant changes in BMI in either of the study groups at either time points.

The final two community level studies were conducted in the USA and examined family based group education interventions that were delivered in school/classroom setting (both targeted approach). One was a randomized controlled trial ${ }^{37}$ that investigated the effects of a 12-week intensive classroom-based physical activity intervention compared with a less intensive activity control group among 92 daughter and mother dyads in a low-income Latino community in the USA. There were no significant differences in maternal $\mathrm{BMI}$ at the end of the 12 -week intervention. The other was a randomized controlled study ${ }^{38}$ to test the effectiveness of a professionally delivered, tailored group education intervention to prevent heart disease in 242 adults in the USA with low-income and low-literacy levels (the Stanford Nutrition Action Programme (SNAP)). It comprised a 6-week, tailored classroom-based intervention followed by a 12-week maintenance intervention. It was compared with nontailored general nutritional group interventions. There were no significant changes in BMI in SNAP compared with the control after either of the intervention periods.

\section{Societal (Environmental $n=1$, macro-policy $n=2$ )}

The 'best available' evidence for the environmental interventions comes from one moderate quality experimental study that took a universal approach and examined a multi-faceted workplace weight prevention intervention. The 'best available' evidence for the macro-level interventions comes from two weak quality observational studies that took a targeted approach and examined effects of the USA Food Stamp Programme, a welfare programme for people with a low-income or with no-income. All three studies were from the USA and two included only women participants.

The environmental intervention study was a cluster randomized controlled trial of 648 (majority female) participants ${ }^{39}$ in the USA that investigated the effects of a 2-year multi-faceted workplace weight prevention intervention on hospital employees. The intervention included a social marketing campaign, interpersonal support groups and environmental strategies to promote physical activity and healthy eating. This included stairway signs, cafeteria signs, farmer's markets, walking groups, challenges, workshops, educational displays, newsletters, project website, project information centre and print materials. The study found that, postintervention participation reduced BMI. However, higher educated participants more likely to prevent weight gain than lower educated participants and so the intervention did not reduce inequalities.

One of the macro-policy intervention studies was a retrospective cohort study ${ }^{40}$ of 5503 USA women that found that a $\$ 2000$ increase in food stamps had no significant effect on weight change unless women were persistently food insecure (the very poorest) whereby a $\$ 2000$ increase was associated with a significant increase in weight $(\beta=7.78, P<0.05)$. A retrospective repeat cross-sectional study ${ }^{41}$ of low-income men and women participants however found no significant effects of the USA Food Stamp Programme on obesity.

\section{DISCUSSION}

What works in reducing inequalities in obesity? for whom? and where?

Evidence of the effectiveness of individual level interventions to reduce inequalities in obesity among adults was only found for tailored weight loss programmes targeted at low-income groups particularly those delivered in primary care settings. These appear to have positive effects on weight outcomes in the short term (up to 9 months). Similarly, we only found evidence of effectiveness for community-based behavioural weight loss interventions and community diet clubs (including work-based ones). However, again these positive effects on obesity-related outcomes were only short term in nature ( 3 months) with no longer term positive effects evident. This is in keeping with previous research into the general effectiveness of obesity-reduction interventions, which has also found short-term benefits of interventions with weight regain in the longer term. ${ }^{42,43}$ The community level workplace studies suggested that longer term positive effects on obesityrelated outcomes require more complex, multi-faceted interventions. This is in keeping with the Foresight obesity review which highlighted the complex multi-factorial nature of inequalities in obesity and therefore the need for sophisticated and longer term interventions to reduce them. ${ }^{44}$ However, in our review, only limited and weak quality evidence was found of more upstream interventions and the results were inconclusive over the longer term. 
It is important to also reflect on 'for whom' and 'where' the interventions were-or more usually were not - effective. The 'best available' international evidence was typically of interventions in the USA perhaps reflecting their more sophisticated approach to experimental design. The 'best available' evidence was also populated by studies with exclusively or majority female participant groups. This is in keeping with the results of general obesity studies where only $10-30 \%$ of participants in weight loss programmes are men. ${ }^{45}$ Often the interventions were targeted at Afro-American or Latin American women. The findings of effectiveness are therefore very much limited to effectiveness among low-income women in the USA. In terms of 'where' interventions were effective then the 'best available' evidence suggested that primary care delivered interventions and those based in community settings (including work places) were the more effective.

\section{Implications for Research}

The nature of the evidence base has a number of implications for public health researchers. Most notably, while we found a very large international evidence base, the quality of the evidence found was largely observational and of moderate to weak quality. These studies did not generally contribute to the 'best available evidence' (with the exception of the macro-policy societal-level interventions). There were few studies of societal-level interventions which might be expected to have more of an impact on the gradient in obesity. ${ }^{16}$ This was particularly the case in terms of the macro-policy level and the few studies that were found did not necessarily have obesity as their main outcome or indeed motivation behind the intervention (for example, the USA food stamp welfare programme was not motivated by a desire to reduce obesity). Similarly, the majority of interventions that were evaluated took a targeted approach to reducing SES inequalities in obesity, with only a minority of studies examining the effects of interventions across the SES gradient. The targeted approach has limitations as even when interventions are effective among lowincome groups they are only able to reduce the health inequalities gap, they have little effect on the wider social gradient. Studies were almost exclusively based on women. The findings of effectiveness are therefore limited to women given that weight loss is embedded in socio-cultural contexts, including those relating to gender. ${ }^{46}$ The focus on women, however, may be explained by the stronger associations between SES and obesity observed in woman. ${ }^{1,4}$ We also found no studies that assessed the cost-effectiveness of interventions and meta-analysis could not be conducted given the heterogeneity of the studies.

Our results show that there is a clear need for more experimental studies of the effectiveness and cost-effectiveness of interventions to reduce inequalities in obesity among both men and women, and especially in terms of macro-level interventions that potentially address the entire gradient.

\section{Implications for Public Health}

Our review has found a large international evidence base but only limited effectiveness of interventions with the potential to reduce SES inequalities in obesity among adults. Most notably, primary care delivered tailored weight loss programmes targeted at individuals from low-income groups and community-based behavioural weight loss interventions and community diet clubs appeared to have some effectiveness-at least in the short term-among low-income women. These interventions may therefore be worth commissioning by those who wish to target services at low-income women or at women in deprived areas. However, to be effective in the longer term, such interventions will need to be of a longer duration and supplemented with subsequent weight maintenance interventions. They may also need to be adapted to be effective among men.

\section{Strengths and Limitations}

This review included an extensive and thorough search of the literature, along with a broad inclusion and exclusion criteria, in order to find the best available evidence at each level of intervention. To ensure reliability, quality check procedures were carried out that included double screening and checking by independent researchers at the title, abstract and full paper screening, data extraction and quality appraisal stages. However, there are also some limitations of this review. We found that the quality appraisal tool, although designed for appraising public health interventions, tended to produce stronger quality scores for those studies following a more clinical model and that some questions were unhelpful. The definitions used for each level of intervention resulted in the majority of interventions being categorized as community level. We also found that some complex interventions containing different elements were difficult to categorize, although we tried to be consistent in our assignment. For this review we did not use ethnicity alone as a proxy measure for SES; therefore, we may have excluded some important studies that did so (for example, ethnicity was often used to indicate SES in the USA), particularly those that explored differential effects by ethnic group.

\section{CONCLUSION}

Our review found a large international evidence base in which the 20 better quality studies suggested that individual, community and societal-level interventions which aim to prevent, reduce or manage obesity at least do not increase inequalities; some universal interventions reduced the gradient in obesity, and many targeted interventions were effective in decreasing obesity among lower socio-economic groups.

Among adults, primary care delivered tailored weight loss programmes targeted at individuals from low-income groups and community-based behavioural weight loss interventions and community diet clubs appeared to have evidence of effectiveness-at least in the short term-among low-income women. More research is needed to determine long term effectiveness, as well as more studies among male populations and of macro-level interventions.

\section{CONFLICT OF INTEREST}

The authors declare no conflict of interest.

\section{ACKNOWLEDGEMENTS}

This project was funded by the National Institute for Health Research (NIHR), the Public Health Research Programme (project number 09/3010/14) and will be published in full by NIHR in 'Public Health Research'. Further information available at: http://www.phr.nihr.ac.uk/funded_projects/obesity.asp. This report presents independent research commissioned by the National Institute for Health Research (NIHR). The views and opinions expressed by authors in this publication are those of the authors and do not necessarily reflect those of the NHS, the NIHR, MRC, CCF, NETSCC, the Public Health Research Programme or the Department of Health. We would like to thank the members of our project steering group for their time and advice throughout the review: Goof Buijs (the Netherlands Institute for Health Promotion and Disease Prevention), Richard Cookson (the University of York), Liam Hughes (the Local Government Improvement and Development), Mike Kelly (the National Institute for Health and Clinical Excellence), Louise Potvin (the University of Montreal) and Martin White (the Newcastle University). We would also like to thank Frances Thirlway for translation services, as well as Jayne Kenworthy and Suzanne Boyd of the Wolfson Research Institute for Health and Wellbeing for their administrative support.

\section{AUTHOR CONTRIBUTIONS}

FCH was responsible for data collection, and contributed to analysis, synthesis. She led the drafting of this article with support from CLB and CDS. CLB was the Principal Investigator and was responsible for overall design, co-ordination and project management. She provided methodological and conceptual direction, and 
contributed to analysis, synthesis and interpretation. J-MC-N contributed to data collection and synthesis. AK contributed to analysis. HJM designed and conducted the searches and contributed to data collection. CDS provided methodological, analysis and synthesis input. All authors contributed to revised successive drafts of this article, and approved the final version and submitted for publication.

\section{REFERENCES}

1 Zhang Q, Wang Y. Trends in the Association between Obesity and Socioeconomic Status in U.S. Adults: 1971 to 2000. Obes Res 2004; 12: 1622-1632.

2 Clarke P, O'Malley PM, Johnston LD, Schulenberg JE. Social disparities in BMI trajectories across adulthood by gender, race/ethnicity and lifetime socio-economic position: 1986-2004. Int J of Epidemiol 2009; 38: 499-509.

3 McLaren L. Socioeconomic status and obesity. Epidemiol Rev 2007; 29: 29-48.

4 El-Sayed A, Scarborough P, Galea S. Unevenly distributed: a systematic review of the health literature about socioeconomic inequalities in adult obesity in the United Kingdom. BMC Public Health 2012; 12: 18.

5 Rohrmann S, Steinbrecher A, Linseisen J, Hermann S, May A, Luan J et al.. The association of education with long-term weight change in the EPICPANACEA cohort. Eur J Clin Nutr 2012; 66: 957-963.

6 Ball K, Crawford D. Socioeconomic status and weight change in adults: a review. Soc Sci Med 2005; 60: 1987-2010.

7 Backholer K, Mannan H, Magliano D, Walls H, Stevenson C, Beauchamp A et al.. Projected socioeconomic disparities in the prevalence of obesity among Australian adults. Aust NZ J Public Health 2012; 36: 557-563.

8 Bambra C, Joyce K, Bellis M, Greatley A, Greengross S, Hughes S et al.. Reducing health inequalities in priority public health conditions: using rapid review to develop proposals for evidence-based policy. J Public Health 2010; 32: 496-505.

9 Bambra C, Joyce KE, Maryon-Davies A. Priority health conditions - Task Group 8 Report to the Strategic Review of Health Inequalities in England post-2010 (Marmot Review). London: UCL, 2009.

10 Robertson A, Lobstein T, Knai C. Obesity and socio-economic groups in Europe: evidence review and implications for action. Rep Eur Comm, pp 1-192, 2007.

11 Whitehead M. A typology of actions to tackle social inequalities in health J Epidemiol Community Health 2007; 61: 473-478.

12 Marmot M, Wilkinson R. Social Determinants Of Health 2nd edn. Oxford University Press: Oxford, 2006.

13 Graham H, Kelly M. Health Inequalities: Concepts, Frameworks And Policy. Health Development Agency: London, 2004.

14 Centre for Reviews and Dissemination. Undertaking systematic reviews of research on effectiveness. CRD's Guidance for Carrying Out or Commissioning Reviews, 2nd edn. York, England, 2001.

15 Higgins J, Green S (eds). Cochrane Handbook for Systematic Reviews of Interventions Version 5.1.0 [updated March 2011]. The Cochrane Collaboration, 2011.

16 Bambra C, Hillier F, Moore H, Cairns-Nagi J, Summerbell C. Tackling inequalities in obesity: a protocol for a systematic review of the effectiveness of public health interventions at reducing socioeconomic inequalities in obesity among adults. Syst Rev 2013; 10: 27.

17 National Institute for Health Public Health Research Programme. PHR - Funded projects 2013. Available at: http://www.phr.nihr.ac.uk/funded_projects/obesity. asp. Accessed 05 December 2013.

18 Effective Public Health Practice Project. Effective Public Health Practice Project Quality Assessment Tool for Quantitative Studies 2009. Available from http:// www.ephpp.ca/tools.html. Accessed 01 September 2011.

19 Davis Martin P, Rhode PC, Dutton GR, Redmann SM, Ryan DH, Brantley PJ. A primary care weight management intervention for low-income AfricanAmerican women. Obesity 2006; 14: 1412-1420.

20 Craigie AM, Macleod M, Barton KL, Treweek S, Anderson AS, WeighWell T. Supporting postpartum weight loss in women living in deprived communities: design implications for a randomised control trial. Eur J Clin Nutr 2011; 65: 952-958.

21 Whittemore R, Melkus G, Wagner J. Translating the diabetes prevention program to primary care: a pilot study. Nurs Res 2009; 58: 2-12.

22 Martin PD, Dutton GR, Rhode PC, Horswell RL, Ryan DH, Brantley PJ. Weight loss maintenance following a primary care intervention for low-income minority women. Obesity 2008; 16: 2462-2467.

23 Jeffery RW, French SA. Preventing weight gain in adults: design, methods and one year results from the Pound of Prevention study. Int J Obes (Lond) 1997; 21: 457-464.

24 Jeffery RW, French SA. Preventing weight gain in adults: The pound of prevention study. Am J Public Health 1999; 89: 747-751.

25 Wing RR, Jeffery RW.. Benefits of recruiting participants with friends and increasing social support for weight loss and maintenance. J Consult Clin Psychol 1999; 67: 132-138.
26 Befort CA, Nollen N, Ellerbeck EF, Sullivan DK, Thomas JL, Ahluwalia JS. Motivational interviewing fails to improve outcomes of a behavioral weight loss program for obese African American women: a pilot randomized trial. $J$ Behav Med 2008; 31: 367-377.

27 Ockene IS, Tellez TL, Rosal MC, Reed GW, Mordes J, Merriam PA et al.. Outcomes of a Latino community-based intervention for the prevention of diabetes: the Lawrence Latino Diabetes Prevention Project. Am J Public Health 2012; 102: 336-342.

28 Reid C, McNeil JJ, Williams F, Powles J. Cardiovascular risk reduction: a randomized trial of two health promotion strategies for lowering risk in a community with low socioeconomic status. J Cardiovasc Risk1995; 2: 155-163.

29 Rickel KA. Response of African-american and Caucasian Women in a Rural Setting to a Lifestyle Intervention for Obesity. ProQuest Information \& Learning: USA, 2008.

30 Faucher MA. From the American College of Nurse-Midwives: promotoras de salud and portion control: a community intervention aimed at weight loss in low-income Mexican-American women. J Midwifery Womens Health 2008; 53: 482.

31 Faucher MA, Mobley J. A community intervention on portion control aimed at weight loss in low-income Mexican American women. J Midwifery Womens Health 2010; 55: 60-64.

32 Auslander W, Haire-Joshu D, Houston C, Williams JH, Krebill H. The short-term impact of a health promotion program for low-income African American women. Res Soc Work Pract 2000; 10: 78-97.

33 Kisioglu AN, Aslan B, Ozturk M, Aykut M, Ilhan I. Improving control of high blood pressure among middle-aged Turkish women of low socioeconomic status through public health training. Croat Med J 2004; 45: 477-482.

34 Baron JA, Schori A, Crow B, Carter R, Mann J. A randomized controlled trial of low carbohydrate and low fat/high fiber diets for weight loss. Am J Public Health 1986; 76: 1293-1296.

35 Erfurt JC, Foote A, Heirich MA. Worksite wellness programs: incremental comparison of screening and referral alone, health education, follow-up counseling, and plant organization. Am J Health Promot 1991; 5: 438-448.

36 Campbell MK, Tessaro I, DeVellis B, Benedict S, Kelsey K, Belton L et al.. Effects of a tailored health promotion program for female blue-collar workers: Health Works for Women. Prev Med 2002; 34: 313-323.

37 Olvera N, Bush JA, Sharma SV, Knox BB, Scherer RL, Butte NF. BOUNCE: a community-based mother-daughter healthy lifestyle intervention for lowincome Latino families. Obesity 2010; 18: S102-S104.

38 Howard-Pitney B, Winkleby MA, Albright CL, Bruce B, Fortmann SP. The Stanford Nutrition Action Program: a dietary fat intervention for low-literacy adults. Am J Public Health 1997; 87: 1971-1976.

39 Lemon SC, Zapka J, Li W, Estabrook B, Rosal M, Magner R et al.. Step ahead: A worksite obesity prevention trial among hospital employees. Am J Prev Med 2010; 38: 27-38.

40 Jones SJ, Frongillo EA. The modifying effects of food stamp program participation on the relation between food insecurity and weight change in women. $J$ Nutr 2006; 136: 1091-1094.

41 Kaushal N. Do food stamps cause obesity? Evidence from immigrant experience. $J$ Health Econ 2007; 26: 968-991.

42 Curioni CC, Lourenco PM. . Long-term weight loss after diet and exercise: a systematic review. Int J Obes Relat Metab Disord 2005; 29: 1168-1174.

43 Wu T, Gao X, Chen M, Van Dam RM. Long-term effectiveness of diet-plus-exercise interventions vs diet-only interventions for weight loss: a meta-analysis. Obes Rev 2009; 10: 313-323.

44 Butland B, Jebb S, Kopelman P, McPherson K, Thomas S, Mardell J et al.. Tackling obesities: Future choices - Project Report. Government Office for Science: London, 2007.

45 Wilkins D. The research base for male obesity: what do we know? In: White A Pettifer M (eds). Hazardous Waist: tackling male weight problems. Radcliffe Publishing Ltd: Abingdon, 2007, pp 3-11.

46 Wolfe B, Smith J. Different strokes for different folks: why overweight men do not seek weight loss treatment. J Eat Disord 2002; 10: 115-124.

(c) (1) $\Theta$ This work is licensed under a Creative Commons Attributioncc. NonCommercial-NoDerivs 3.0 Unported License. The images or other third party material in this article are included in the article's Creative Commons license, unless indicated otherwise in the credit line; if the material is not included under the Creative Commons license, users will need to obtain permission from the license holder to reproduce the material. To view a copy of this license, visit http://creativecommons.org/licenses/by-nc-nd/3.0/ 